\title{
A new family of matrix product states with Dzyaloshinski-Moriya interactions
}

\author{
Marzieh Asoudeh \\ Department of Physics, Shahid Beheshti University, GC \\ 19839-63113, Tehran, Iran
}

\begin{abstract}
We define a new family of matrix product states which are exact ground states of spin 1/2 Hamiltonians on one dimensional lattices. This class of Hamiltonians contain both Heisenberg and Dzyaloshinskii-Moriya interactions but at specified and not arbitrary couplings. We also compute in closed forms the one and two-point functions and the explicit form of the ground state. The degeneracy structure of the ground state is also discussed.
\end{abstract}

PACS Numbers: 03.67.HK, 05.40.Ca 


\section{Introduction}

In the past few years, a lot of interest has been attracted to the subject of matrix product states $[1,3,3,4,4,5,6,17,8,9$, . There has been a revival of interest in this subject [10, 11, 12, 13, 14, 15, 16] due to the advances in quantum information theory [17] and the techniques developed in this field. Quite recently the method of matrix product states has been used for obtaining new models of many body systems for which an exact ground state can be defined and its many-body properties like the entanglement between various sites can be calculated in closed form [18, 19, 20, 21, 22, 23, 24, 25, 26]. This is interesting in view of the fact that in some works, transitions in entanglement behaviour has turned out [30, 31] to be a good signal of quantum phase transitions [29].

The reason for this high level of interest is the complementary role that the fields of condensed matter physics and quantum information play in investigation of many body systems. The matrix product formalism [32, 33] is one of the subjects which lies at the borderline of these two subjects. As is well known, in this formalism, one starts from proposed states whose expansion coefficients are the trace of product of given matrices. While for numerical investigations, i.e. the density matrix renormalization group (DMRG), one usually starts from large dimensional matrices, to simulate ground states of given Hamiltonians, in the approach which is used for finding exactly solvable models, one starts from low dimensional matrices and finds family of Hamiltonians for which these states are exact ground states. This is the approach which has been used in many of the works in the past few years [1, 2, 3, 4, 15, 18, 19, 20, 21, 22, 24, 23, 25, 26].

In a recent work [26], we classified all spin 1/2 matrix product states which arise from two-dimensional matrices and showed that some of them can undergo phase transitions. Doing the same kind of classification for higher dimensional matrices turns out to be a formidable task. A general classification in effect requires classification of solutions of a system of non-linear equations, which obviously is extremely difficult. However it is possible to define new models by starting with special classes of matrices which have nice algebraic properties. Such properties allow us to bypass the above-mentioned difficult problem. The price that we will pay is that we will obtain only restricted family of models and not all models. However if we start from a nice algebra, it is usually possible to end at physically interesting models and by the subsequent analysis of these models beyond the matrix product formalism, many useful information can be obtained for such models which are otherwise difficult to obtain. We will later see explicit examples of this method.

Following this method, in this paper we will introduce one such family of matrix product states which is based on a simple algebra

$$
X Z=\omega Z X
$$

where $\omega$ is a root of unity. We use a finite dimensional representation of this algebra in which $Z$ and $X$ are represented by generalized Pauli operators. Everything in 
the model, from the state and its parent Hamiltonian (i.e. the Hamiltonian which has the matrix product state as its ground state) to the one- and two-point functions depend on the dimension of the representation. We will show that while for $D=2$ dimensional representation of the algebra, the parent Hamiltonian, is a one parameter of Heisenberg spin chain, for $D \neq 2$ dimensional representation the Hamiltonian contains both Heisenberg and Dzyaloshinskii-Moriya interactions [27, 28, but for specified values of couplings which depend on the dimensional representation $D$.

It is to be noted that the matrix product state is by construction a translationinvariant state and in some cases, it happens that this state is a sum of two ground states each of which breaks the translation symmetry. This is the case with MajumdarGhosh [34, 35] model [20]. Here we will see a similar phenomena, where the matrix product state is the sum of many ground states each of which breaks the translation symmetry of the original Hamiltonian.

The calculation of the one- and two-point functions is an important step in this analysis. While such a calculation is straightforward in the formalism of matrix product formalism, a close scrutiny of their structure will give clues as to the whole or part of the ground space structure and by following these clues we can obtain valuable information about the degeneracy structure of the ground state.

The structure of this paper is as follows. In section (2) we give a brief introduction to matrix product states, in section (3) we introduce the representation of the algebra (11) and construct the family of matrix product states and derive the parent Hamiltonian, in section (55) we calculate the one and two point functions. Finally we end up with a discussion.

\section{A brief introduction to matrix product states}

First let us review the basics of matrix product states. Consider a homogeneous ring of $N$ sites, where each site describes a $d$-level state. The Hilbert space of each site is spanned by the basis vectors $|i\rangle, \quad i=0, \cdots d-1$. A state

$$
|\Psi\rangle=\sum_{i_{1}, i_{2}, \cdots i_{N}} \psi_{i_{1} i_{2} \cdots i_{N}}\left|i_{1}, i_{2}, \cdots, i_{N}\right\rangle
$$

is called a matrix product state if there exists $D$ dimensional complex matrices $A_{i} \in$ $C^{D \times D}, \quad i=0 \cdots d-1$ such that

$$
\psi_{i_{1}, i_{2}, \cdots i_{N}}=\frac{1}{\sqrt{Z}} \operatorname{tr}\left(A_{i_{1}} A_{i_{2}} \cdots A_{i_{N}}\right),
$$

where $Z$ is a normalization constant given by

$$
z=\operatorname{tr}\left(E^{N}\right)
$$


and

$$
E:=\sum_{i=0}^{d-1} A_{i}^{*} \otimes A_{i} .
$$

Here we are restricting ourselves to translationally invariant states, by taking the matrices to be site-independent.

Let $O$ be any local operator acting on a single site. Then we can obtain the one-point function on site $k$ of the chain $\langle\Psi|O(k)| \Psi\rangle$ as follows:

$$
\langle\Psi|O(k)| \Psi\rangle=\frac{\operatorname{tr}\left(E^{k-1} E_{O} E^{N-k}\right)}{\operatorname{tr}\left(E^{N}\right)}
$$

where

$$
E_{O}:=\sum_{i, j=0}^{d-1}\langle i|O| j\rangle A_{i}^{*} \otimes A_{j} .
$$

The n-point functions can be obtained in a similar way. For example, the two-point function $\langle\Psi|O(k) O(l)| \Psi\rangle$ can be obtained as

$$
\langle\Psi|O(k) O(l)| \Psi\rangle=\frac{\operatorname{tr}\left(E_{O}(k) E_{O}(l) E^{N}\right)}{\operatorname{tr}\left(E^{N}\right)}
$$

where $E_{O}(k):=E^{k-1} E_{O} E^{-k}$. Note that this is a formal notation which allows us to write the n-point functions in a uniform way, it does not require that $E$ is an invertible matrix. Also by considering the permutation operator $P$ defined as

$$
P|\alpha \beta\rangle=|\beta \alpha\rangle
$$

and using equation(5) we see that $E^{*}=P E P$ and since $P^{2}=P$ we find that $Z^{*}=Z$, meaning that the normalization is real as it should be. Also for a Hermitian operator $O$ in equation (6) the one point function $\langle\Psi|O(k)| \Psi\rangle$ will be real. The same reasoning applies for n-point functions.

\subsection{The Parent Hamiltonian}

Given a matrix product state, the reduced density matrix of $k$ consecutive sites is given by

$$
\rho_{i_{1} \cdots i_{k}, j_{1} \cdots j_{k}}=\frac{\operatorname{tr}\left(\left(A_{i_{1}}^{*} \cdots A_{i_{k}}^{*} \otimes A_{j_{1}} \cdots A_{j_{k}}\right) E^{N-k}\right)}{\operatorname{tr}\left(E^{N}\right)} .
$$

The null space of this reduced density matrix includes the solutions of the following system of equations

$$
\sum_{j_{1}, \cdots, j_{k}=0}^{d-1} c_{j_{1} \cdots j_{k}} A_{j_{1}} \cdots A_{j_{k}}=0 .
$$

Given that the matrices $A_{i}$ are of size $D \times D$, there are $D^{2}$ equations with $d^{k}$ unknowns. Since there can be at most $D^{2}$ independent equations, there are at least $d^{k}-D^{2}$ 
solutions for this system of equations. Thus for the density matrix of $k$ sites to have a null space it is sufficient that the following inequality holds

$$
d^{k}>D^{2} .
$$

Let the null space of the reduced density matrix be spanned by the orthogonal vectors $\left|e_{\alpha}\right\rangle, \quad\left(\alpha=1, \cdots s, \geq d^{k}-D^{2}\right)$. Then we can construct the local hamiltonian acting on $k$ consecutive sites as

$$
h:=\sum_{\alpha=1}^{s} \mu_{\alpha}\left|e_{\alpha}\right\rangle\left\langle e_{\alpha}\right|
$$

where $\mu_{\alpha}$ 's are positive constants. These parameters together with the parameters of the vectors $\left|e_{i}\right\rangle$ inherited from those of the original matrices $A_{i}$, determine the total number of coupling constants of the Hamiltonian. If we call the embedding of this local Hamiltonian into the sites $l$ to $l+k$ by $h_{l, l+k}$ then the full Hamiltonian on the chain is written as

$$
H=\sum_{l=1}^{N} h_{l, l+k} .
$$

The state $|\Psi\rangle$ is then a ground state of this hamiltonian with vanishing energy. The reason is as follows:

$$
\langle\Psi|H| \Psi\rangle=\operatorname{tr}(H|\Psi\rangle\langle\Psi|)=\sum_{l=1}^{N} \operatorname{tr}\left(h_{l, l+k} \rho_{l, l+k}\right)=0,
$$

where $\rho_{l, k+l}$ is the reduced density matrix of sites $l$ to $l+k$ and in the last line we have used the fact that $h$ is constructed from the null eigenvectors of $\rho$ for $k$ consecutive sites. Given that $H$ is a positive operator, this proves the assertion.

In view of the above introduction, we have a clear receipe for constructing matrix product states and a family of parent Hamiltonians. First one chooses the range of interaction $k$ and then choose appropriate matrices, throwing away all spurious degrees of freedom by appropriate transformations $A_{i} \longrightarrow S A_{i} S^{-1}$ and reducing further the degrees of freedom by imposing symmetries. In this way one ends with a reasonable set of matrix product states, which hopefully may have applications in description of real physical systems. In the following section, we want to study a particular family of such states arising from the simple algebra 1 .

\section{The model}

In this paper we specify the auxiliary matrices $A_{0}$ and $A_{1}$ as $D$ dimensional generalization of spin (Pauli) operators in $x$ and $z$ directions. This is a $\mathrm{D}$ dimensional representation of the algebra 1. Hereafter, for convenience, we denote $A_{0}$ and $A_{1}$ respectively by $X$ and $Z$. In the so called computational basis for qudits, spanned by the orthonormal vectors $\{|0\rangle,|1\rangle, \cdots|n\rangle\}$ and we have

$$
X=\sum_{n=0}^{d-1}|n+1\rangle\langle n|,
$$


and

$$
Z=\sum_{n=0}^{D-1} \omega^{n}|n\rangle\langle n|
$$

where $\omega=e^{\frac{2 \pi i}{D}}$.

One finds from (16 and 17) that

$$
Z X=\omega X Z
$$

As explained in section 2, equation (5), the transfer matrix for our model has the following simple form

$$
E=X \otimes X+\bar{Z} \otimes Z
$$

The matrix $E$ plays a central role for calculating the normalization of the ground state and also in determining all the correlation functions. We need to find the spectrum of this matrix. The right and left eigenvectors of $E$ turn out to be

$$
\begin{gathered}
\left|\psi_{k}(r)\right\rangle:=\frac{1}{\sqrt{D}} \sum_{n=0}^{D-1} \omega^{-r n}|n, n+k\rangle, \\
\left\langle\psi_{k}(r)\right|:=\frac{1}{\sqrt{D}} \sum_{n=0}^{D-1} \omega^{r n}\langle n, n+k|,
\end{gathered}
$$

both of which correspond to the eigenvalue

$$
\lambda_{k}(r)=\omega^{k}+\omega^{r}, \quad k, r=0,1, \cdots D-1 .
$$

It is easily checked that $\left\langle\psi_{k}(r) \mid \psi_{k^{\prime}}\left(r^{\prime}\right)\right\rangle=\delta_{k, k^{\prime}} \delta_{r, r^{\prime}}$.

Also we need to know $Z=\operatorname{tr}\left(E^{N}\right)$, the normalization constant which from (22) turns out to be

$$
Z_{D}(N)=\sum_{k, r=0}^{D-1}\left(\omega^{r}+\omega^{k}\right)^{N}
$$

where we have re-labeled the partition function as $Z_{D}(N)$ to emphasize its dependence on the number of sites $N$ and the dimension of the representation $D$. From the binomial expansion, we find

$$
Z_{D}(N)=\sum_{r, k=0}^{D-1} \sum_{l=0}^{N}\left(\begin{array}{c}
N \\
l
\end{array}\right) \omega^{r l} \omega^{k(N-l)}
$$

Summing over $r$ and $k$ and noting that $\sum_{r=0}^{D-1} \omega^{r l}=D \delta_{l, m D}$ and $\sum_{k=0}^{D-1} \omega^{k(N-l)}=$ $D \delta_{N-l, m^{\prime} D}$ where $m$ and $m^{\prime}$ are integers we find that $Z_{D}(N)$ is nonzero only when $N$ is a multiple of $D$. Hereafter we assume that this is the case, that is we set $N \longrightarrow N D$ 
in all the equations. The physical necessity of this requirement will become clear when we discuss the explicit form of the ground states. With this modification, we find the

$$
Z_{D}(D N)=D^{2} \sum_{l=0}^{N}\left(\begin{array}{c}
D N \\
D l
\end{array}\right) .
$$

This is a sum of $N$ terms, where $N$ is the number of sites and as such is not a close expression. However we can use an identity and rewrite it as a sum of $D$ terms which is much simpler. To this end we consider the following binomial identity

$$
\left(1+\omega^{s}\right)^{N D}=\sum_{l=0}^{N D}\left(\begin{array}{c}
N D \\
l
\end{array}\right) \omega^{s l},
$$

and sum over both sides for $s=0,1, \cdots D-1$ to obtain

$$
\begin{aligned}
\sum_{s=0}^{D-1}\left(1+\omega^{s}\right)^{N D} & =\sum_{s=0}^{D-1} \sum_{l=0}^{N D}\left(\begin{array}{c}
N D \\
l
\end{array}\right) \omega^{s l} \\
& =\sum_{l=0}^{N D}\left(\begin{array}{c}
N D \\
l
\end{array}\right) \sum_{s=0}^{D-1} \omega^{s l}
\end{aligned}
$$

However, since $\omega^{D}=1$, the sum over $s$ in the right hand side is non-vanishing only when $l$ is a multiple of $D$ and in that case it will be equal to $D$. Therefore we find that

$$
Z_{N D}=D \sum_{i=0}^{D-1}\left(1+\omega^{s}\right)^{N D}
$$

In contrast to (25), this is a sum over $D$ terms which is independent of the system size and obviously is much simpler.

For example for $D=2$ and for $D=3$ we have respectively

$$
Z_{2 N}=2^{2 N+1}
$$

and

$$
Z_{3 N}=3\left(2^{3 N}+2(-1)^{N}\right)
$$

We will now consider the parent Hamiltonian, for which the matrix product state is an exact ground state.

\subsection{The Parent Hamiltonian}

To find the parent Hamiltonian, we use the relation $\sum C_{i j} A_{i} A_{j}=0$ which in view of (1) takes the form 


$$
C_{00} X^{2}+C_{01} X Z+C_{10} Z X+C_{11} Z^{2}=0 .
$$

For $D \neq 2$ the operators $X^{2}$ and $Z^{2}$ are independent. However in any dimension $Z X=\omega X Z$ and hence the vanishing of the left hand side gives the following relations on the coefficients

$$
C_{00}=C_{11}=0, \quad C_{01}+\omega C_{10}=0 .
$$

According to (32), these equations define the null space of the density matrix of two adjacent sites. This null space is spanned by only one vector, which we denote by $|e\rangle$,

$$
|e\rangle=|10\rangle-\omega|01\rangle \text {. }
$$

Thus the local Hamiltonian is given by

$$
h_{k, k+1}=|e\rangle\left\langle\left. e\right|_{k, k+1}=(|10\rangle-\omega|01\rangle)(\langle 10|-\bar{\omega}\langle 01|)_{k, k+1} .\right.
$$

Writing the above expression in terms of the Pauli operators, we find that

$$
h_{k, k+1}=\frac{1}{2}\left(I-\sigma_{k}^{z} \sigma_{k+1}^{z}\right)-\omega \sigma_{k}^{+} \sigma_{k+1}^{-}-\bar{\omega} \sigma_{k}^{-} \sigma_{k+1}^{+} .
$$

After a simple re-scaling $H \longrightarrow 2 H$ and neglecting an additive constant, the full Hamiltonian becomes

$$
H=\sum_{k=1}^{N} \cos \frac{2 \pi}{D}\left(\sigma_{k}^{x} \sigma_{k+1}^{x}+\sigma_{k}^{y} \sigma_{k+1}^{y}\right)-\sigma_{k}^{z} \sigma_{k+1}^{z}+\sin \frac{2 \pi}{D}\left(\sigma_{k}^{x} \sigma_{k+1}^{y}-\sigma_{k}^{y} \sigma_{k+1}^{x}\right) .
$$

This is the general form of the Hamiltonian $H$ when we use the $D \neq 2$ dimensional representation of the algebra (11). Therefore this Hamiltonian contains both Heisenberg and Dzyaloshinskii-Moriya interaction [27, 28], but with specific and not arbitrary couplings.

In case we use the $D=2$ dimensional representation, a different Hamiltonian will be obtained, since in this case we have $X^{2}=Z^{2}=I$ and the null-space condition (37) will have a different solution. In this null-space condition (37) turns out to be

$$
\left(C_{00}+C_{11}\right) I+\left(C_{01}-C_{10}\right) Z X=0,
$$

and the null space is spanned by two vectors, namely

$$
\left|e_{1}\right\rangle=|00\rangle-|11\rangle, \quad\left|e_{2}\right\rangle=|01\rangle+|10\rangle .
$$

This larger null space means that the Hamiltonian has now two arbitrary coupling constants, and by a re-scaling $H \longrightarrow 2 H$, the Hamiltonian takes the form

$$
H=\sum_{i=1}^{N}\left(J_{1}-J_{2}\right)\left(\sigma_{k}^{z} \sigma_{k+1}^{z}-\sigma_{k}^{x} \sigma_{k+1}^{x}\right)+\left(J_{1}+J_{2}\right)\left(I+\sigma_{k}^{y} \sigma_{k+1}^{y}\right) .
$$

In this case the Dzyaloshinskii-Moriya interaction is absent. Note the Hamiltonian 39 cannot be obtained from 36 in a certain limit. Up to now the parent Hamiltonian has been obtained for both types of representations of the matrix product algebra (37). The next step will be to calculate the correlation functions. We limit ourselves to one and two-point functions. 


\section{One and two-point correlation functions}

The matrix product formalism allows a straightforward calculation of correlation functions. According to (7), the matrix product operators corresponding to the spin observables $\sigma_{x}, \sigma_{y}$ and $\sigma_{z}$ are

$$
\begin{aligned}
& E_{x}:=X \otimes Z+Z \otimes X, \\
& E_{y}:=-i X \otimes Z+i Z \otimes X, \\
& E_{z}:=X \otimes X-Z \otimes Z .
\end{aligned}
$$

For finding the average of Pauli operators in each site of the chain we use equation (66). As explained in section (22), for finite systems, this requires a diagonalization of the transfer matrix $E$, while in the thermodynamic limit even this problem simplifies to the determination of the largest eigenvalue of the transfer matrix. In some cases, i.e. for the two-dimensional representation of the algebra (11), one can even proceed in an alternative and very simple way. Therefore we organize the content of this section in two separate subsections, where we deal separately, using different methods, with 2-dimensional and higher dimensional representations of the matrix product algebra.

\subsection{Two-dimensional representation}

Let us start by noting that for the two-dimensional representation, we have $X^{2}=$ $Z^{2}=I$ and $Z X=-X Z$. The same relations hold with $X \longrightarrow Y$. These relations and the expression for the transfer operator E given in (19) leads to the simple relations

$$
E_{x} E=E_{y} E=E_{z} E=0
$$

from which we immediately obtain that

$$
\left\langle\sigma_{k}^{x}\right\rangle=\left\langle\sigma_{k}^{y}\right\rangle=\left\langle\sigma_{k}^{z}\right\rangle=0
$$

It is obvious from the relations (41) that any correlation function containing $\sigma_{x}$ and $\sigma_{y}$ operators on non-adjacent sites will also vanish. For adjacent sites we have to calculate traces of operators like $E_{x}^{2} E^{N-2}, E_{y}^{2} E^{N-2}$ and $E_{x} E_{y} E^{N-2}$. It is simply obtained from (40) that

$$
E_{x}^{2} E=0, \quad E_{y}^{2} E=-4 E, \quad E_{z}^{2} E=0,
$$

which leads to

$$
\left\langle\sigma_{k}^{x} \sigma_{k+1}^{x}\right\rangle=0 \quad\left\langle\sigma_{k}^{y} \sigma_{k+1}^{y}\right\rangle=-4 \frac{\operatorname{tr} E^{2 N-2}}{\operatorname{tr} E^{2 N}}=-1, \quad\left\langle\sigma_{k}^{z} \sigma_{k+1}^{z}\right\rangle=0,
$$

where we have used equation (40). The last relation implies that the ground state

is one in which the the spins of adjacent sites are aligned in the $y$ directions but in 
opposite senses. There are two such states and the matrix product state, which by construction should be translation invariant, is the sum of these two states, namely

$$
|\Psi\rangle=\frac{1}{\sqrt{2}}\left(\left|+_{y},-_{y}\right\rangle^{\otimes N}+\left|-_{y},+_{y}\right\rangle^{\otimes N}\right),
$$

where $\left| \pm_{y}\right\rangle:=\frac{1}{\sqrt{2}}\left(\begin{array}{c}1 \\ \pm i\end{array}\right)$ are the two eigenstates of the $\sigma^{y}$ operator. By using the relations

$$
\sigma^{z}\left| \pm_{y}\right\rangle=|\mp y\rangle, \quad \sigma^{x}\left| \pm_{y}\right\rangle= \pm i\left|\mp_{y}\right\rangle, \quad \sigma^{y}\left| \pm_{y}\right\rangle= \pm\left| \pm_{y}\right\rangle,
$$

one can readily check that the two states in the superposition of $|\psi\rangle$ are actually annihilated by the Hamiltonian, so they are the ground states of the Hamiltonian. In fact a simple way to see this, is to rotate all the spins in the odd-numbered sites by an angle $\pi$ around the $z$-axis. Under such a rotation, the Hamiltonian (39) transforms to

$$
H^{\prime}=\sum_{i=1}^{N}\left(J_{1}-J_{2}\right)\left(\sigma_{k}^{z} \sigma_{k+1}^{z}+\sigma_{k}^{x} \sigma_{k+1}^{x}\right)+\left(J_{1}+J_{2}\right)\left(I-\sigma_{k}^{y} \sigma_{k+1}^{y}\right) .
$$

or equivalently by adding and subtracting terms of the form $\sigma_{k}^{y} \sigma_{k+1}^{y}$, it transforms to

$$
H^{\prime}=\sum_{i=1}^{N}\left(J_{1}-J_{2}\right)\left(\vec{\sigma}_{k} \cdot \vec{\sigma}_{k+1}\right)-2 J_{1} \sigma_{k}^{y} \sigma_{k+1}^{y}
$$

where $\vec{\sigma}_{k} \cdot \vec{\sigma}_{k+1}=\sigma_{k}^{x} \sigma_{k+1}^{x}+\sigma_{k}^{y} \sigma_{k+1}^{y}+\sigma_{k}^{z} \sigma_{k+1}^{z}$ and we have dropped a total additive constant. In case that $0<J_{1}<J_{2}$, the first term is a ferromagnetic interaction which tends to align all the spins in one single direction, the direction is determined by the second $y-y$ interaction. Hence we have a doubly degenerate ground state of the form $\left|\Phi_{+}\right\rangle:=|y+\rangle^{\otimes 2 N}$ and $\left|\Phi_{-}\right\rangle:=|y-\rangle^{\otimes 2 N}$ for $H^{\prime}$. Rotating back all the odd-numbered spins by $\pi$ around the $z$ axis gives the two ground states of $H$ as expressed in (45), where their sum has been obtained as a matrix product state. Thus in this case the MPS formalism guides us through the complete degenerate structure of the ground state. However if $J_{1}>J_{2}>0$, then the first term in $H^{\prime}$ is an anti-ferromagnetic interaction with a highly degenerate complex structure. In this case the MPS only gives a very small part of the spectrum.

\subsection{Higher dimensional representations}

We now turn to higher dimensional representation where the Hamiltonian contains a Dzyialoshinski-Moriya interaction. In this case the commutation relations do not allow a simple calculation of the correlation functions for finite systems. Therefore we calculate the correlations directly in the thermodynamic limit. In this limit, only the largest eigenvalue and eigenvector of the transition operator $E$ will survive the limit $N \longrightarrow \infty$ and one finds that 


$$
\left\langle O_{k}\right\rangle=\frac{1}{\lambda_{\max }}\left\langle\lambda_{\max }\left|E_{O}\right| \lambda_{\max }\right\rangle
$$

and

$$
\left\langle O_{k} O_{k+1}\right\rangle=\frac{1}{\lambda_{\max }^{2}}\left\langle\lambda_{\max }\left|E_{O}^{2}\right| \lambda_{\max }\right\rangle
$$

where $\lambda_{\max }$ and $\left|\lambda_{\max }\right\rangle$ are the largest eignevalue of $E$ and its corresponding eigenvector. From (22) we know that the largest eigenvalue of $E$ is 2 corresponding to the generalized Bell state $\left|\Psi_{00}\right\rangle=\frac{1}{\sqrt{D}} \sum_{n=0}|n, n\rangle$. For the one-point functions we need the matrix elements of the operators $E_{x}, E_{y}$, and $E_{z}$ on this Bell state. It is obvious from (40) that $\left\langle\psi_{00}\left|E_{x}\right| \psi_{00}\right\rangle=\left\langle\psi_{00}\left|E_{x}\right| \psi_{00}\right\rangle=0$. For $E_{z}$ we find that

$$
\begin{aligned}
\left\langle\psi_{00}\left|E_{z}\right| \psi_{00}\right\rangle & =\frac{1}{D} \sum_{n, m=0}^{D-1}\langle m, m|X \otimes X-\bar{Z} \otimes Z| n, n\rangle \\
& =\frac{1}{D} \sum_{n, m=0}^{D-1}\left(\delta_{m, n+1}-\delta_{m, n}\right)=0 .
\end{aligned}
$$

Therefore we find

$$
\left\langle\sigma_{x}\right\rangle=\left\langle\sigma_{y}\right\rangle=\left\langle\sigma_{z}\right\rangle=0 .
$$

We now proceed to calculate the two-point functions for adjacent sites. To do this we need the following matrix elements which are readily obtained from (40) after simple manipulations:

$$
\begin{aligned}
\left\langle\psi_{00}\left|E_{x}^{2}\right| \psi_{00}\right\rangle & =\omega+\omega^{-1}=2 \cos \frac{2 \pi}{D}, \\
\left\langle\psi_{00}\left|E_{y}^{2}\right| \psi_{00}\right\rangle & =\omega+\omega^{-1}=2 \cos \frac{2 \pi}{D}, \\
\left\langle\psi_{00}\left|E_{z}^{2}\right| \psi_{00}\right\rangle & =0
\end{aligned}
$$

and

$$
\begin{aligned}
& \left\langle\psi_{00}\left|E_{x} E_{y}\right| \psi_{00}\right\rangle=i\left(\omega-\omega^{-1}\right)=-2 \sin \frac{2 \pi}{D} \\
& \left\langle\psi_{00}\left|E_{y} E_{x}\right| \psi_{00}\right\rangle=-i\left(\omega-\omega^{-1}\right)=2 \sin \frac{2 \pi}{D} .
\end{aligned}
$$

Moreover all the other quadratic matrix elements turn out to vanish. From the above matrix elements, one can easily obtain the correlation functions from (49) as follows:

$$
\left\langle\sigma_{k}^{x} \sigma_{k+1}^{x}\right\rangle=\left\langle\sigma_{k}^{y} \sigma_{k+1}^{y}\right\rangle=\frac{1}{2} \cos \frac{2 \pi}{D} \quad, \quad\left\langle\sigma_{k}^{z} \sigma_{k+1}^{z}\right\rangle=0
$$

and

$$
\left\langle\sigma_{k}^{x} \sigma_{k+1}^{y}\right\rangle=-\left\langle\sigma_{k}^{y} \sigma_{k+1}^{x}\right\rangle=\frac{1}{2} \sin \frac{2 \pi}{D}
$$




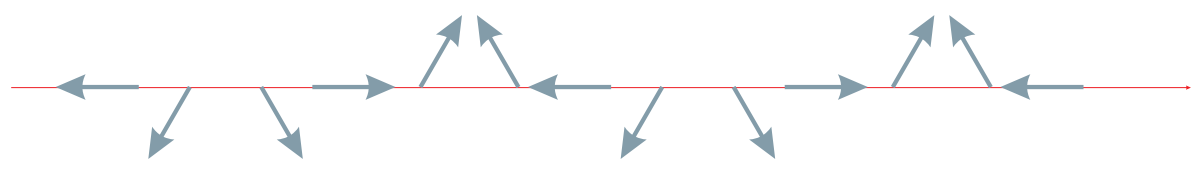

Figure 1: (Color online) One of the product states $|\chi\rangle$ (for $\mathrm{D}=6)$. The matrix product state is a superposition of $D$ such states, each shifted by one site with respect to the previous one.

All the other two-point correlation functions vanish for nearest neighbor sites. The above results can be written in the following compact form:

$$
\left\langle\vec{\sigma}_{k} \cdot \vec{\sigma}_{k+1}\right\rangle=\cos \frac{2 \pi}{D} \quad\left\langle\vec{\sigma}_{k} \times \vec{\sigma}_{k+1}\right\rangle=-\sin \frac{2 \pi}{D} \hat{z} .
$$

From these correlation functions along with the result (45) which we obtained for the explicit form of the ground state for the two-dimensional representations, we can guess an explicit form for the ground state of the Hamiltonian (36). We will later check the validity of our guess by direct calculations. The ground state (45), turned out to be a superposition of two product states, each one of them was nothing but a juxtaposition of spins in opposite directions. In fact each of the two product states are ground states of the Hamiltonian separately, and the matrix product state superposes them to ensure its own translational invariance. The same thing may happen here, that is we may find product states each of which is a ground state of the Hamiltonian and the matrix product state is a superposition of them. Inspired by the form of the ground states for the $\mathrm{D}=2$ representation, we construct a product state which gives the same form of correlation functions as in (56). This construction is a generalization of the states in (45). Let

$$
|\phi\rangle:=\sin \phi\left|+_{x}\right\rangle+\cos \phi\left|{ }_{y}\right\rangle,
$$

where $\left|+_{x}\right\rangle$ and $\left|+{ }_{y}\right\rangle$ are the spin states in the positive $x$ and $y$ directions respectively. We now form the following product state on $D$ consecutive sites (instead of two sites in the $D=2$ case)

$$
|\chi(\phi)\rangle:=|\phi\rangle\left|\phi-\frac{2 \pi}{D}\right\rangle\left|\phi-\frac{4 \pi}{D}\right\rangle \cdots\left|\phi-\frac{2(D-1) \pi}{D}\right\rangle .
$$

Each consecutive spin has been rotated anti-clockwise by an angle of $\frac{2 \pi}{D}$ around the $z$ axis, in analogy with the $D=2$ dimensional case. That is, the spins in each block of $D$ consecutive sites are distributed regularly around a circle in the $x-y$ plane. It is obvious that for any such block state, comprising $D$ consecutive sites, the average of spin operator in any direction should vanish, that is

$$
\left\langle\sigma^{a}\right\rangle_{\chi(\phi)}:=\frac{1}{D} \sum_{i=0}^{D-1}\left\langle\chi(\phi)\left|\sigma_{i}^{a}\right| \chi(\phi)\right\rangle=0, \quad a=x, y, z .
$$

The reason is nothing but the regular distribution of the spin states $|\phi\rangle$ over the circle. The same type of reasoning, which can be supported by direct and explicit 
calculations, shows the validity of the two-point correlations (56) for any block state of the above form.

We can extend such a state to the whole lattice as a non-translation invariant $|\chi(\phi)\rangle^{\otimes N}$, which obviously has the same kinds of correlations as in (56). The matrix product state, being translation invariant is nothing but the following superposition

$$
\left|\Psi_{D}\right\rangle:=|\chi(0)\rangle^{\otimes N}+\left|\chi\left(\frac{2 \pi}{D}\right)\right\rangle^{\otimes N}+\left|\chi\left(\frac{4 \pi}{D}\right)\right\rangle^{\otimes N} \cdots+\left|\chi\left(\frac{2(D-1) \pi}{D}\right)\right\rangle^{\otimes N} .
$$

This formula for the MPS ground state generalized the explicit formula (45) to higher dimensional representations of the algebra.

It is instructive to know the origin of the character of the ground state (59). Let us proceed as in the $D=2$ case and rotate the spins individually, but this time, according to a different pattern. Consider the local Hamiltonian

$$
h_{k, k+1}=\cos \frac{2 \pi}{D}\left(\sigma_{k}^{x} \sigma_{k+1}^{x}+\sigma_{k}^{y} \sigma_{k+1}^{y}\right)-\sigma_{k}^{z} \sigma_{k+1}^{z}+\sin \frac{2 \pi}{D}\left(\sigma_{k}^{x} \sigma_{k+1}^{y}-\sigma_{k}^{y} \sigma_{k+1}^{x}\right)
$$

and rotate the spin at site $k+1$ by an angle $\theta=-\frac{2 \pi}{D}$ around the $z$ axis. Under this transformation, the spin operators transform as follows:

$$
\begin{aligned}
& \sigma_{k+1}^{z} \longrightarrow \sigma_{k+1}^{z} \\
& \sigma_{k+1}^{x} \longrightarrow-\cos \theta \sigma_{k+1}^{x}+\sin \theta \sigma_{k+1}^{y} \\
& \sigma_{k+1}^{y} \longrightarrow \sin \theta \sigma_{k+1}^{x}-\cos \theta \sigma_{k+1}^{y}
\end{aligned}
$$

Under this transformation, it is readily seen that $h_{k, k+1}$ transforms to an isotropic Heisenberg ferromagnet, $h_{k, k+1}^{H e i s}$

$$
h_{k, k+1}^{H e i s}=-\left(\sigma_{k}^{x} \sigma_{k+1}^{x}+\sigma_{k}^{y} \sigma_{k+1}^{y}+\sigma_{k}^{z} \sigma_{k+1}^{z}\right) .
$$

It is well known that the ground state of the Heisenberg Hamiltonian $H^{\text {Heis }}=$ $\sum_{k} h_{k, k+1}^{H e i s}$ is the state in which all the spins align in one direction, and since the Hamiltonian has rotational symmetry, the ground state consists of a whole spin multiplet, which is the multiplet with the Highest spin. For a lattice with $N D$ sites, this multiplet has the spin $\frac{N D}{2}$ whose top state is $\left|\frac{N D}{2}, \frac{N D}{2}\right\rangle=\left|z_{+}\right\rangle^{\otimes N D}$. The other states are obtained by the action of the angular momentum operator $L_{-}:=\sum_{k=1}^{N D} \sigma_{k}^{-}$on this top state. That is, the other un-normalized ground states are

$$
\left|\frac{N D}{2}, m\right\rangle=L_{-}^{\otimes\left(\frac{N D}{2}-m\right)}\left|\frac{N D}{2}, \frac{N D}{2}\right\rangle .
$$

Acting on these states by the inverse rotations $\bigotimes_{k} R_{z}\left(\frac{2 k \pi}{D}\right)$ we obtain the ground states of the original Hamiltonian in the form 


$$
\Psi_{m}:=\left[\bigotimes_{k} R_{z}\left(\frac{2 k \pi}{D}\right)\right] L_{-}^{\otimes\left(\frac{N D}{2}-m\right)}\left|\frac{N D}{2}, \frac{N D}{2}\right\rangle .
$$

The ground states in $|\chi(\phi)\rangle^{\otimes N}$ from which the matrix product state (59) is constructed are only part of the ground space of the Hamiltonian. As expected in the matrix product formalism one starts from one single state, but a careful analysis of that single state and its correlation functions, assisted by some guesswork and physical analogies, can unravel the whole degeneracy structure of the Hamiltonian.

\section{Discussion}

We have started from a simple matrix product algebra, namely $X Z=\omega Z X$ where $\omega^{D}=1$ and proceeded to obtain the properties of the matrix product state which corresponds to this algebra. There is a distinct difference between the cases $D=2$ and $D \neq 2$. In the former case, the model describes a system of spins on a line with Heisenberg type interaction but with prescribed couplings, in the latter case, the model describes both a Heisenberg and a Dzyaloshinski-Morya (DM) interaction. In both cases we have been able to calculate the one and two point functions and from the insight that these functions have provided, we have been able to obtain a more thorough understanding of these models, including the degeneracy structure of their ground state. One possible line of expansion of this work is to start from a Heisenberg ferromagnetic chain of arbitrary spins in a magnetic field. If now we rotate in a periodic pattern the magnetic fields and demand, similar to the work of Kurman et al [36], that all the cross terms except the Heisenberg and the DM interactions vanish, we will end up with a new form of exactly solved Heisenberg model in which DM interactions are also present. The difficulty with this approach is that if we start with a uniform magnetic field, the final magnetic fields will not be uniform anymore, due to the periodic rotations. On the other hand if we start with a non-uniform magnetic field and demand that the final magnetic field will be uniform along the chain, then the spectrum of the original Hamiltonian will be very difficult to find. We hope to overcome these difficulties in our future work.

Acknowledgements I would like to thank V. Karimipour for many instructive discussions and valuable comments and also for a critical reading of the manuscript.

\section{References}

[1] A. Klumper, A. Schadschneider and J. Zittartz, J. Phys. A (1991) L293; Z. Phys. B, 87 (1992) 281; Europhys. Lett., 24 (1993) 293.

[2] H. Niggemann, and J. Zittartz, J. Phys. A: Math. Gen. 31, 9819-9828 (1998).

[3] E. Bartel, A. Schadschneider and J. Zittartz, Eur. Phys. Jour. B, 31, 2, 209-216 (2003). 14 
[4] M. A. Ahrens, A. Schadschneider, and J. Zittartz, Europhys. Lett. 59 6, 889 (2002).

[5] A. K. Kolezhuk and H. J. Mikeska, Phys. Rev. Lett. 80, 2709 (1998); Int. J. Mod. Phys. B, 12, 2325-2348 (1998).

[6] A. K. Kolezhuk, H. J. Mikeska, and Shoji Yamamoto, Phys. Rev. B 55, R3336 R3339 (1997).

[7] H. Niggemann, A. Klumper, and J. Zittartz, Eur. Phys. J. B 13, 15 (2000).

[8] H. Niggemann, A. Klumper, and J. Zittartz, Z. Phys. B 104, 103 (1997).

[9] A. Klumper, S. Matveenko, and J. Zittartz, Z. Phys. B 96, 401 (1995).

[10] F. Verstraete, J. J. Garcia-Ripoll, and J. I. Cirac, Phys. Rev. Lett. 93, 207204 (2004).

[11] F. Verstraete, J. I. Cirac, Phys. Rev. B 73, 094423 (2006); T. J. Osborne, Phys. Rev. Lett. 97, 157202 (2006); M. B. Hastings, Phys. Rev. B 73, 085115 (2006).

[12] G. Vidal, Phys. Rev. Lett. 91, 147902 (2003).

[13] M. M. Wolf, G. Ortiz, F. Verstraete and I. Cirac, Phys. Rev. Lett. 97, 110403 (2006).

[14] F. Verstraete, M. A. Martin-Delgado, and J. I. Cirac, Phys. Rev. Lett. 92, 087201 (2004); F. Verstraete, M. Popp, and J. I. Cirac, Phys. Rev. Lett. 92, 027901 (2004); F. Verstraete, J. I. Cirac, J. I. Latorre, E. Rico, and M. M. Wolf, Phys. Rev. Lett. 94 140601(2005).

[15] F. Verstraete, D. Porras, J. I. Cirac, Phys. Rev. Lett. 93, 227205 (2004).

[16] J. M. Roman, G. Sierra, J. Dukelsky, and M. A. Martn-Delgado, J. Phys. A: Mathematical and General, 31, 48, 9729-9759(1998).

[17] M. A. Nielsen, and I. L. Chuang;Quantum computation and quantum information, Cambridge University Press, Cambridge, 2000.

[18] M. Asoudeh, V. Karimipour, and A. Sadrolashrafi, Phys. Rev. A 76, 012320 (2007).

[19] M. Asoudeh, V. Karimipour and A. Sadrolashrafi, Phys. Rev. B, 75, 224427 (2007).

[20] M. Asoudeh, V. Karimipour, and A. Sadrolashrafi, Phys. Rev. A 76, 012320 (2007).

[21] S. Alipour, V. Karimipour and L. Memarzadeh, Phys. Rev. A 75, 052322 (2007). 
[22] S. Alipour, V. Karimipour, and L. Memarzadeh, Eur. Phys. J. B 62, 159-169 (2008).

[23] S. Alipour, S. Baghbanzadeh, and V. Karimipour, C Europhysics Letters (EPL), $84(2008) 67006$.

[24] V. Karimipour and L. Memarzadeh, Phys. Rev. B 77, 094416 (2008). 15

[25] A. Trebedi and I. Bose, Phys. Rev. A 75, 042304(2007).

[26] M. Asoudeh, Physica A; 389, 8, 1555-1564 (2010).

[27] I. Dzyaloshinskii, J. Phys. Chem. Solids 4, 241 (1958).

[28] T. Moriya, Phys. Rev 120, 91 (1960).

[29] S. Sachdev, Quantum Phase Transitions (Cambridge University Press, Cambridge, 1999).

[30] A. Osterloh, L. Amico, G. Falci, and R. Fazio, Nature 416, 608 (2002).

[31] T. J. Osborne and M. A. Nielsen, Phys. Rev. A 66, 032110 (2002).

[32] M. Fannes, B. Nachtergaele and R. F. Werner, Commun. Math. Phys. 144, 443 (1992).

[33] I. Affleck, T. Kennedy, E. H. Lieb, H. Tasaki, Commun.Math. Phys. 115, 477 (1988);

[34] C. K. Majumdar and D. P. Ghosh, J. Math. Phys. 10 (1969)1388.

[35] C. K. Majumdar and D. P. Ghosh, J. Math. Phys. 10 (1969)1399.

[36] J. Kurmann, H. Thomas and G. Muller, Physica 112 A 235(1982). 